\title{
Lentiviral-Mediated RNA Interference against TGF-Beta Receptor Type II in Renal Epithelial and Fibroblast Cell Populations In Vitro Demonstrates Regulated Renal Fibrogenesis That Is More Efficient than a Nonlentiviral Vector
}

\author{
Tao Yang, ${ }^{1,2}$ Bing Zhang, ${ }^{3}$ Betty K. Pat, ${ }^{1,4}$ Ming Q. Wei, ${ }^{5}$ and Glenda C. Gobe ${ }^{1}$ \\ ${ }^{1}$ Centre for Kidney Disease Research, School of Medicine, The University of Queensland at Princess Alexandra Hospital, \\ Brisbane, QLD 4102, Australia \\ ${ }^{2}$ Discipline of Pathology, School of Medicine, University of Western Sydney, Sydney, NSW 2751, Australia \\ ${ }^{3}$ Department of Employment, Economic Development and Innovation, Brisbane, QLD 4003, Australia \\ ${ }^{4}$ Department of Medicine, University of Alabama, Birmingham, AL 35487, USA \\ ${ }^{5}$ Division of Molecular and Gene Therapies, School of Medical Science, Griffith University, Gold Coast, QLD 4222, Australia
}

Correspondence should be addressed to Ming Q. Wei, m.wei@griffith.edu.au

Received 29 March 2010; Revised 5 July 2010; Accepted 18 August 2010

Academic Editor: G. S. Stein

Copyright (C) 2010 Tao Yang et al. This is an open access article distributed under the Creative Commons Attribution License, which permits unrestricted use, distribution, and reproduction in any medium, provided the original work is properly cited.

\begin{abstract}
Background. Lentiviral constructs reportedly can integrate into the genome of non-dividing, terminally differentiated cells and dividing cells, for long-term gene expression. This investigation tested whether a third generation lentiviral-mediated small interfering RNA (siRNA) delivered into renal epithelial and fibroblast cells against type II transforming growth factor-beta receptor (siRNA-TBRII) could better attenuate renal fibrogenesis in comparison with a non-lentiviral construct. Methods. HIV-derived lentiviral and non-lentiviral constructs were used to transfect cells with siRNA-TBRII or siRNA-EGFP control. Human embryonic kidney (HEK-293T), renal epithelial cells (NRK-52E) and renal fibroblasts (NRK-49F) were transfected and gene silencing quantified (fluorescence microscopy, Western blotting, fluorescence-activated cell sorting). Renal fibrogenesis was assessed using extracellular matrix protein synthesis (fibronectin and collagen-III; Western immunoblot), and $\alpha$-smooth muscle actin ( $\alpha$ SMA) was analysed as a marker of fibroblast activation and epithelial-to-mesenchymal transdifferentiation (EMT). Results. Lentiviral-mediated siRNA-TBRII significantly suppressed TBRII expression in all cell lines, and also significantly suppressed renal fibrogenesis. In comparison with the non-lentiviral construct, lentiviral-mediated siRNA-TBRII produced stronger and more persistent inhibition of collagen-III in NRK-49F cells, fibronectin in all renal cell lines, and $\alpha$-SMA in renal epithelial cells. Conclusions. Lentiviral vector systems against TBRII can be delivered into renal cells to efficiently limit renal fibrogenesis by sequence-specific gene silencing.
\end{abstract}

\section{Background}

Tubulointerstitial fibrosis is an almost invariable finding in the chronically diseased kidney, irrespective of the underlying disease or the originating compartment. The degree of fibrosis, determined by the relative interstitial volume caused by accumulation of extracellular matrix (ECM), is an important predictor of organ prognosis and kidney excretory function [1]. Renal tubulointerstitial fibrosis is believed to be the final common pathway for nearly all forms of kidney disease that progress towards end-stage renal disease [2].
Transforming growth factor- $\beta$ (TGF- $\beta$ ), a protein regulator of cell growth and differentiation, is one of the primary mediators that induces accumulation of ECM in renal fibrogenesis $[3,4]$. TGF- $\beta$ increases the production and deposition of ECM proteins, reduces matrix degradation through decreased proteinase production and increased production of matrix degradation inhibitors, and stimulates synthesis of ECM protein receptors [5]. Upregulation of TGF- $\beta$ is a universal finding in virtually every type of chronic kidney disease, in animal models and in humans [4]. In vitro, TGF- $\beta$ alone can stimulate mesangial cells and interstitial 
fibroblasts to produce ECM and tubular epithelial cells to undergo epithelial-to-mesenchymal transformation (EMT) to become matrix-producing fibroblast-like cells. Expression of exogenous TGF- $\beta$, either via gene delivery in vivo or in transgenic mice, causes renal fibrosis [6]. TGF- $\beta$ binds to specific receptors on most cells including renal epithelial and fibroblast cells and then initiates a signal cascade that results in production of profibrotic cytokines and inflammatory mediators. The type I and type II signalling receptors mediate the biological actions of TGF- $\beta$. The extracellular domain of the type II receptor (TBRII) binds TGF- $\beta$, causing the formation of heteromeric complexes $[7,8]$. TBRII then transphosphorylates the type I receptor, activates its kinase, and initiates downstream signalling. In this respect, the TBRII appears to be essential for the biological activity of TGF- $\beta$ signalling pathway [9], and its inhibition or modification is considered as a promising therapeutic strategy to inhibit renal fibrosis.

Modulation of TGF- $\beta$ action on ECM suppresses tissue fibrosis $[2,10]$. For example, biological inhibition of TGF$\beta$ protein with neutralising antibodies [11], decorin [12, 13], and soluble TBRII-IgG Fc chimera [14] suppressed the accumulation of ECM in models of renal fibrosis. Other studies have shown that expression of soluble TBRII could effectively block TGF- $\beta$ signalling in vitro and in vivo, using various means of delivery $[15,16]$. However, the methods used in previous studies have therapeutic limitations because the protein or gene is rapidly degraded by enzymes after administration in vivo $[17,18]$. The short-term duration of TGF- $\beta$ signalling inhibition is a major problem to be solved.

Lentiviral vectors can infect non-dividing cells, can be pseudotyped with retroviral envelopes, and so have a broad cell tropism, have no toxic effect, and have stable gene expression due to viral genome integration into cell chromosomes [19]. Despite having many of the same characteristics, retroviral vector-mediated gene therapies or nonviral vector-mediated gene deliveries have a limited or transient effect and can infect only dividing cells [19]. Melding the lentiviral vector-mediated gene delivery and the powerful tool of RNA inhibition (RNAi) could potentially provide targeted long-term gene silencing. RNAi is a mechanism for sequence-specific, posttranscriptional gene silencing triggered by double-strand RNA (dsRNA; referred to as small interfering RNA, silencing RNA or siRNA), which targets the degradation of complementary mRNAs [20]. The siRNA mediators of RNAi typically consist of 19-23 nucleotide RNA duplexes. During RNAi, the introduction of siRNA by transfection into a diverse range of organisms and cell types causes degradation of the complementary mRNA, thereby silencing gene expression.

Because of the ability of lentiviral vectors to deliver transgenes into tissues that are considered resistant to stable genetic manipulation, like well-differentiated adult renal cell populations with limited levels of mitosis, new possibilities for gene therapy are opening. Gusella et al. [21] successfully transduced a 1st-generation lentiviral vector into mouse kidneys. The current study used a cell culture model of renal fibrosis to compare the efficiency to limit renal fibrogenesis and the durability of expression of a lentiviral versus a nonlentiviral versus a lentiviral-delivered siRNA to TBRII (siRNA-TBRII).

\section{Methods}

2.1. Cell Culture. Rat renal epithelial (NRK-52E, ATCC CRL1571) and fibroblast (NRK-49F, ATCC CRL1570) cell lines were selected for comparison of fibrogenesis. Human embryonic kidney- (HEK-) 293T cells, a derivative of the HEK-293 cell line (ATCC CRL1573) were selected for plasmid transfection and lentiviral vector transduction. An enhanced green fluorescent protein (EGFP) stably expressing HEK-293T cell line (S7 cells, established at Dr. Ming Wei's laboratory, Department of Medicine, Prince Charles Hospital, University of Queensland, Brisbane, Australia) was used for transfection to evaluate the silencing effect on EGFP by RNAi.

NRK-49F and NRK-52E cells were maintained routinely in Dulbecco's modified Eagle's medium (DMEM) supplemented with $10 \%$ bovine calf serum (BCS), 1,000 units per millilitre $(\mathrm{U} / \mathrm{mL})$ penicillin, and $1,000 \mu \mathrm{g} / \mathrm{mL}$ streptomycin (BioWhittaker, Edward Keller Pty Ltd, Silverwater, Australia). For HEK-293T cells and the EGFP stably expressing S7 cells, DMEM was supplemented with $10 \%$ fetal calf serum (FCS). Cells were grown in a humidified atmosphere of $95 \%$ air and $5 \%$ carbon dioxide at $37^{\circ} \mathrm{C}$ in a tissue culture incubator. The original cell seeding density was of $2-5 \times 10^{5}$ cells per $\mathrm{mL}$. Cells were grown for 24-48 hours prior to treatment or until just subconfluent. Prior to treatment, all cells were washed twice in serum-free (SF) DMEM containing antibiotics only, to remove any traces of serum growth factors or supplements.

2.2. PCR Cloning and Plasmid Preparations. A 444 bp extracellular ectodomain including the critical soluble TBRII TGF- $\beta$ binding domain comprising the amino acid DNA sequence from $284 \mathrm{bp}$ to $727 \mathrm{bp}$ (targeting the sequence between starting codon and the beginning of hydrophobic trans-membrane spanning domain) of Rattus norvegicus TBRII mRNA (Genbank s67770) [22] was generated by reverse transcriptase PCR from normal rat kidney cDNA using a QIAGEN Taq PCR core kit. The $444 \mathrm{bp}$ DNA sequence of the TBRII gene was ligated into the pDrive vector (QIAGEN, Australia) at a molar ratio of 5-10 times more PCR product DNA than pDrive cloning vector DNA. The orientation of PCR product in the recombinant plasmid was confirmed by restriction enzyme digestion. After enzyme digestion, the recombinant plasmid pDrive-TBRII with the PCR insert of the 444bp soluble domain of the TBRII gene was mini-preped using a GenElute Plasmid Miniprep Kit (Sigma-Aldrich, Australia) and maxi-preped for a larger amount of plasmid production using a Plasmid Maxi Kit (QIAGEN, Australia). The constructed pDrive-TBRII with its PCR insert was further confirmed by DNA sequencing at the Australian Genome Research Facility (Gehrmann Laboratories, University of Queensland, http://www.agrf.org.au/). 
2.3. Preparation of RNAi Expression Plasmids. Two complementary oligonucleotides necessary to create the hairpin insert for pPlasRi cloning vectors were designed following a software siRNA Wizard v2.5 available on line at http://www.sirnawizard.com/. The sequences of custom-designed oligonucleotides were as follows: TBRII RNAi short hairpin (sh) insert "A" targeting the first $100 \mathrm{bp}$ starting from ATG start codon: 5'-GAT CCC C ACGTTCCCAAGTCGGTTAA T TCA AGA GA TTAACCGACTTGGGAACGT TTT TTG GAA A-3' and $5^{\prime}$ - TCGA TT TCC AAA AA ACGTTCCCAAGTCGGTTAA TCT CTT GAA TTAACCGACTTGGGAACGT GG G-3', insert "B" targeting the last $100 \mathrm{bp}$ of codons of TBRII: 5 -GAT CCC C AGATTCCAGAAGACGGCTC T TCA AGA GA GAGCCGTCTTCTGGAATCT TTT TTG GAA A-3' and 5' -TCGA TT TCC AAA AA AGATTCCAGAAGACGGCTC TCT CTT GAA GAGCCGTCTTCTGGAATCT GG G-3'. The 19-nucleotide TBRII target sequences are underlined in these sequences. Two single-strand oligonucleotides synthesised for EGFP and used as experimental control siRNA were provided by Dr. Ming Q. Wei (one of the authors). All oligos were purchased in a lyophilised desalted form from Sigma Genosys Australia (Castle Hill, Australia). A pair of oligonucleotides was annealed using the methods previously described [23]. After annealing of two-single stranded oligos into double-stranded inserts, siTBRII and siEGFP were ligated to linearised pPlasRi plasmid (provided by Dr. Ming Q. Wei, one of the authors) that contains the human H1 RNA Polymerase III promoter after BglII and SalI digestion. Compatible enzyme sequences were synthesized at both ends of annealed oligos. Both siTBRII inserts showed efficient silencing effect at the proof of concept experiment, and the pair targeting the fist $100 \mathrm{bp}$ was used in this study. In addition, pPlasRi without any insertion of oligonucleotide (empty pPlasRi) was used as a negative control.

2.4. Production of Lentiviral RNAi Constructs. The thirdgeneration replication-defective HIV-1-based lentiviral vector pHIV-CS (7488 bp) was provided by Dr. Ming Q. Wei, one of the authors. Both lentiviral RNAi constructs (pLentiTBRII and pLenti-EGFP) were constructed by cloning the pHIV-CS backbone with siRNA inserts derived from pPlasRi-RBRII or pPlasRi-EGFP by BamHI and XhoI digestion, in which the RNA promoter was also transferred to the lentiviral RNAi plasmids.

2.5. Lentiviral Construct Transfection In Vitro. For in vitro transfection, $1 \times 10^{5}$ cells were seeded per well in 12 -well plates 16-18 hours before the experiment, by which time the cells reached $75-80 \%$ confluence. Plasmids DNA of $2 \mu \mathrm{g}$ with a linear PEI derivative (ExGen 500 MBI Fermentas, Hanover MD, USA), at N/P ratio of $1: 6$ to $1: 9$, were incubated for 10 minutes at room temperature and then added to the wells. The transfection medium containing plasmid DNA was replaced 8 hours later with fresh growth medium and cells were further incubated for 16 hours or longer for up to 72 hours as required.
2.6. FACS Quantification of EGFP Expression. EGFP gene expression was analysed by direct fluorescence using a confocal microscope (Queensland Institute for Medical Research, Brisbane), and images were digitised. Fluorescence-activated cell sorting (FACS; Becton Dickinson and Co, Franklin Lakes NJ, USA) was performed using 10,000 fixed cells at various intervals after transfection to quantify the number and the mean fluorescence intensity of cells positive for EGFP. Untransfected HEK-293T and S7 cells were used to determine the cutoff values. Gene silencing effectiveness was evaluated by direct visualization under a fluorescent microscope (in the stably EGFP-expressing S7 cells which were transfected by EGFP RNAi plasmids) and quantified by FACS. Immunofluorescence (IF) microscopy or Western immunoblot was used for protein analysis of TBRII in NRK49F and NRK-52E cells which were transfected by TBRII RNAi plasmids.

2.7. Immunofluorescence. For IF staining of TBRII on transfected HEK-293T cells, cells on coverslips were washed and fixed, and then nonspecific binding was blocked with $3 \%$ bovine serum albumin in Tris-buffered saline with Tween20 (BSA in TBST) for $1 \mathrm{hr}$ at room temperature. Coverslips were incubated with primary antibody against TBRII (TGF $\beta$ RII, 1:10, Santa Cruz) for 1 hour at room temperature (RT). Sections were washed $3 \times 30$ minutes in TBST, then incubated with a fluorescent secondary antibody mixture containing an FITC-labelled goat antirabbit IgG (Molecular Probes, Oregon, USA) at a dilution of $1: 200$ in $1 \%$ BSA in TBST for 1 hour at RT in a dark cupboard. Coverslips were washed $3 \times 30$ minutes in TBST, then mounted onto glass slides using DAKO antifade fluorescence mounting medium (DAKO, Australia), and allowed to set overnight in the dark at $4^{\circ} \mathrm{C}$ or over 2-3 hours at RT prior to confocal fluorescence microscopy.

2.8. Western Immunoblot. Cells were disrupted in ice-cold cell lysis buffer $(50 \mathrm{mM}$ Tris-Cl at $\mathrm{pH} 7.5,150 \mathrm{mM} \mathrm{NaCl}$, $1 \%$ Nonidet P-40, $0.1 \%$ sodium dodecyl sulphate (SDS), $25 \mathrm{mM}$ sodium fluoride, and $0.5 \mathrm{M}$ ethylenediamine tetraacetic acid) containing protease and phosphatase inhibitors ( $100 \mu \mathrm{g} / \mathrm{mL}$ phenylmethylsulfonyl chloride, $20 \mu \mathrm{g} / \mathrm{mL}$ leupeptin, $20 \mu \mathrm{g} / \mathrm{mL}$ aprotinin, and $1 \mathrm{mM}$ sodium orthovanadate, all Sigma-Aldrich products) using a tissue homogenizer. Cell debris was removed by centrifugation at $16000 \mathrm{~g}$ for 15 minutes at $4^{\circ} \mathrm{C}$. Protein concentration was determined in each extract by a Bradford protein assay (Bio-Rad Pty Ltd, Sydney, NSW, Australia) and spectroscopy at $595 \mathrm{~nm} .30 \mu \mathrm{g}$ of total protein were loaded onto $12 \%$ SDS polyacrylamide gel, run at $100 \mathrm{~V}$ with a set current of $60 \mathrm{mAmps}$ for 1.75 hours, and then transferred to a polyscreen polyvinylidene difluoride membrane (Perkin Elmer, Melbourne, Australia) using a Bio-Rad Mini Protean 3 unit (Bio-Rad, Australia). Nonspecific binding of antibodies to the membrane was blocked by incubation in $4 \%$ skim milk powder in TBST buffer (blotto) for 1 hour with continuous rocking. Antibodies were added to blotto or $0.1 \%$ BSA in TBST (primary 
antibody TGF $\beta$ RII Mouse mAb) and incubated with membranes overnight at $4^{\circ} \mathrm{C}$ on a rocking platform. Blots were washed repeatedly (4-5 times, 5-10 minutes each) in TBST and then incubated with the corresponding Horseradish peroxidase- (HRP-) conjugated secondary antibody (rabbit$\alpha$-mouse IgG-HRP) diluted in either $4 \%$ blotto or $0.1 \%$ BSA in TBST for 1 hour at RT on a rocking platform. Membranes were subjected to a second stage of vigorous and stringent washes (4-5) in TBST containing 0.1\% Igepal CA-630, each for 5-minute duration. Membranes were incubated with SuperSignal West Pico chemiluminescent substrate (Pierce Biotechnology, Rockford, IL, USA) for 2 minutes with vigorous shaking. Protein bands were detected using enhanced chemiluminescence (ECL) imaging onto Xray film (Kodak X-Omat AR-5 or Fuji Rx XR Film) and scanned using a Hewlett-Packard ScanJet 3200C at 300 dpi. Scion Image software $(\mathrm{v} \beta 4.0 .2)$ was used to quantify the density of protein bands minus local background in arbitrary densitometry units. All protein bands were normalised against actin, or membranes were stained with Coomassie Brilliant Blue (Sigma-Aldrich: catalogue number B7920) to verify equal protein loading of lanes. Cell extract positive controls from antibody suppliers were routinely used for immunoblots.

2.9. Statistical Analysis. Data were analysed using standard statistical methods, linear regression, Students, $t$-test, oneway analysis of variance (ANOVA), and Tukey's, Dunnett's multiple comparison, or Bonferroni posttest using Graphpad Prism (version 3.0) and Stata (version 8.0) statistical software. Most data are presented in graphic form as the means \pm SEM. Significance was assessed at $P<.05$.

\section{Results}

3.1. RNAi Expression Constructs for TBRII and EGFP. TBRII and EGFP RNAi expression constructs were constructed first, followed by the lentiviral RNAi construct. EGFP was used as a reporter gene. The RNAi effect was first tested by transfection of the expression plasmids in vitro before moving onto Lenti-RNAi plasmid construction. Once the gene silencing effect was confirmed, RNAi cassettes together with the upstream RNA promoter were transferred into a third-generation HIV-1 based lentiviral plasmid. Two single-stranded oligonucleotides synthesised for TBRII and EGFP siRNA were annealed to be double-stranded inserts. Constructed RNAi expression plasmids pPlasRi-TBRII and pPlasRi-EGFP were confirmed by restriction digestion.

3.2. Inhibition of EGFP by RNAi Expression Construct and Lentiviral RNAi Construct In Vitro. EGFP RNAi expression construct pPlasRi-EGFP and lentiviral RNAi construct pLentiRi-EGFP were transfected into S7 cells, facilitated by the cationic polymer PEI. Silencing of EGFP expression by RNAi was evaluated by counting numbers of green fluorescent cells by FACS analysis at 24 hours, 48 hours, and 72 hours post transfection. Empty pPlasRi and pLentiRi constructs without RNAi insert were used as negative controls. These results are demonstrated in Figure 1. There was $78.28 \%, 69.37 \%$, and $40.09 \%$ inhibition of EGFP expression $(P<.001)$ as measured by FACS at 24, 48, and 72 hours, respectively, post transfection of pPlasRi-EGFP into S7 cells. The negative control group did not show EGFP inhibition. The RNAi expression construct pPlasRi-EGFP gene silencing effect was however transient, peaking at 24 hours, and then starting to diminish. In comparison, persistent EGFP inhibition up to 72 hours was observed postlentiviral RNAi construct pLentiRi-EGFP transfection, with an $84.17 \%$, 91.57\%, and 95.91\% decrease of EGFP expression achieved at 24, 48, and 72 hours, respectively, and EGFP silencing effect by lentiviral construct increased with time. There was no significant difference in gene silencing effect when comparing the transfection of the two constructs at 24 hours. The lentiviral RNAi construct, however, demonstrated significant advantages in posttranscriptional gene silencing than the expression construct at 48 and 72 hours. No EGFP inhibition was observed in the negative control group.

3.3. Posttranscriptional Sequence-Specific Gene Silencing of TBRII by RNAi In Vitro. Having proven successful EGFP gene silencing by RNAi-expressing and lentiviral-RNAi constructs in S7 cells, RNAi gene silencing of TBRII was evaluated in renal epithelial and fibroblast cell lines (NRK$52 \mathrm{E}$ and NRK-49F) in vitro. An embryonic renal cell line HEK-293T was also tested. Inhibition of ECM accumulation in renal cells as a result of downregulation of TGF- $\beta$ signalling by RNAi was investigated.

3.4. TBRII Gene Suppression in HEK-293T by RNAi. TBRII RNAi expression construct pPlasRi-TBRII and lentiviral RNAi construct pLentiRi-TBRII were transfected into cultured HEK-293T cells, facilitated by transfection agent PEI. Inhibition of TBRII was examined by FITC-fluorescence immunohistochemistry and Western blot at 24, 48, and 72 hours. Empty pPlasRi and pLentiRi constructs without RNAi insert were used as negative controls. Inhibition of TBRII expression was observed after both pPlasRi-TBRII and pLentiRi-TBRII transfection. TBRII inhibition in both experimental groups persisted for up to 72 hours posttransfection. pLentiRi-TBRII construct transfection showed a significantly stronger inhibition of TBRII when compared to pPlasRi-TBRII at 72 hours $(P<.01)$. Gene silencing effect of lentiviral RNAi construct increased with time $(P<.01)$. These results are shown in Figure 2.

3.5. TBRII Gene Suppression by RNAi in Renal Epithelial and Fibroblast Cells. Having confirmed the RNAi effect in HEK-293T cells post-transfection of both RNAi expression construct and lentiviral RNAi construct, gene silencing of TBRII was tested in renal epithelial (NRK-52E) and fibroblast (NRK-49F) cells. Expression of TBRII protein was evaluated by Western blot analysis and semiquantified by densitometry at 24,48 , and 72 hours post-transfection of pPlasRi-TBRII and pLentiRi-TBRII. There was a significant decrease of TBRII expression in both cell lines at all tested 

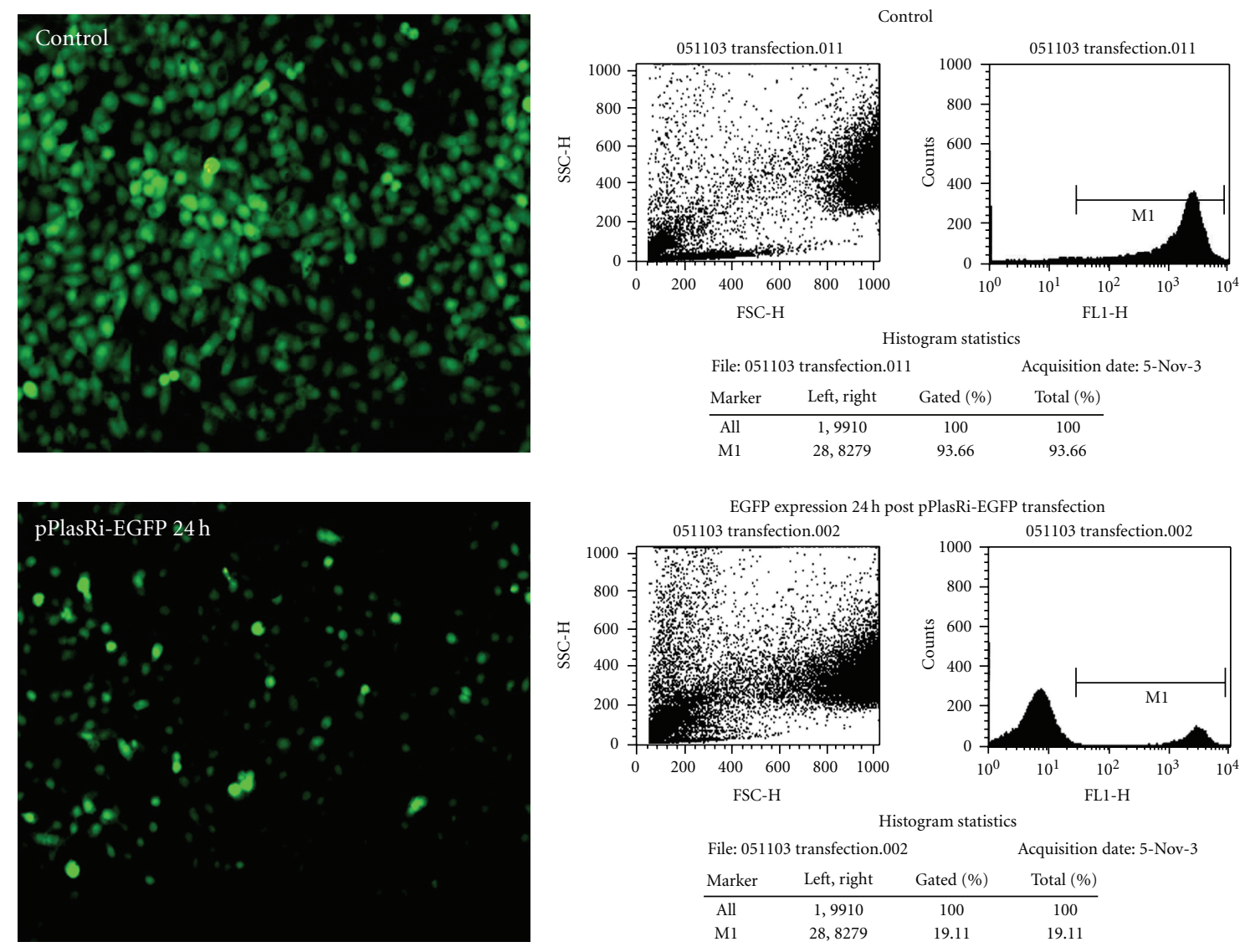

File: 051103 transfection.002

Acquisition date: 5-Nov-3

\begin{tabular}{cccc} 
Marker & Left, right & Gated (\%) & Total (\%) \\
\hline All & 1,9910 & 100 & 100 \\
M1 & 28,8279 & 19.11 & 19.11
\end{tabular}
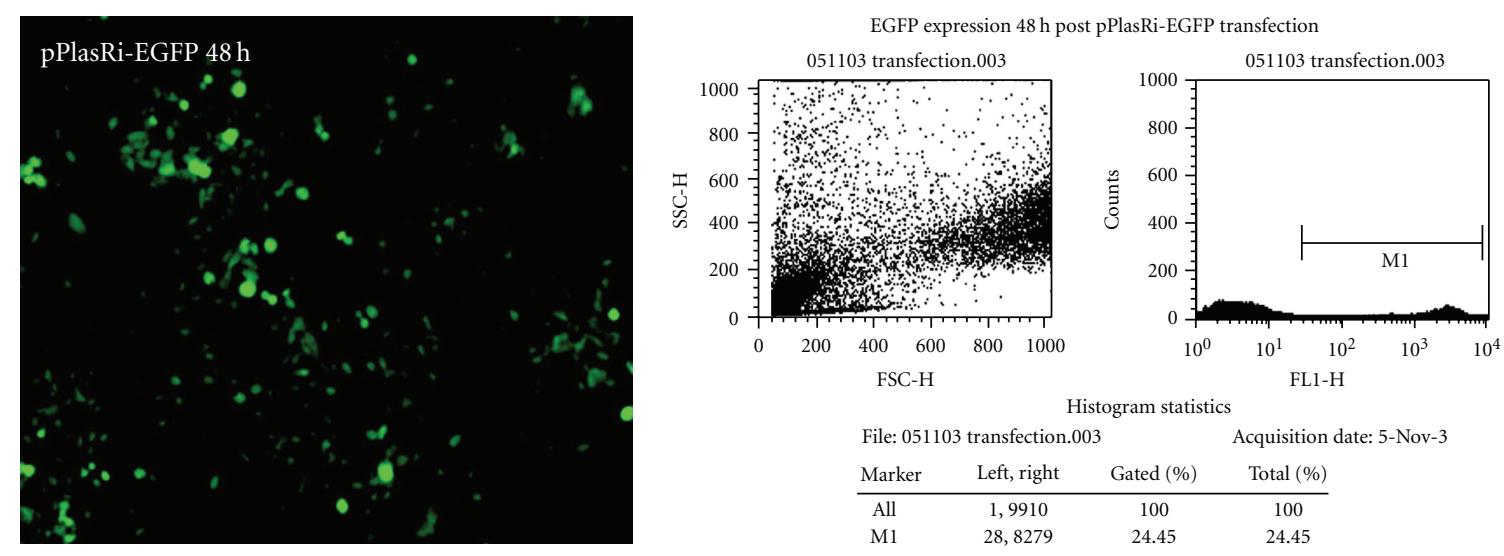

$$
\text { FSC-H }
$$

Histogram statistics

File: 051103 transfection.003 Acquisition date: 5-Nov-3

\begin{tabular}{cccc} 
Marker & Left, right & Gated $(\%)$ & Total $(\%)$ \\
\hline All & 1,9910 & 100 & 100 \\
M1 & 28,8279 & 24.45 & 24.45
\end{tabular}
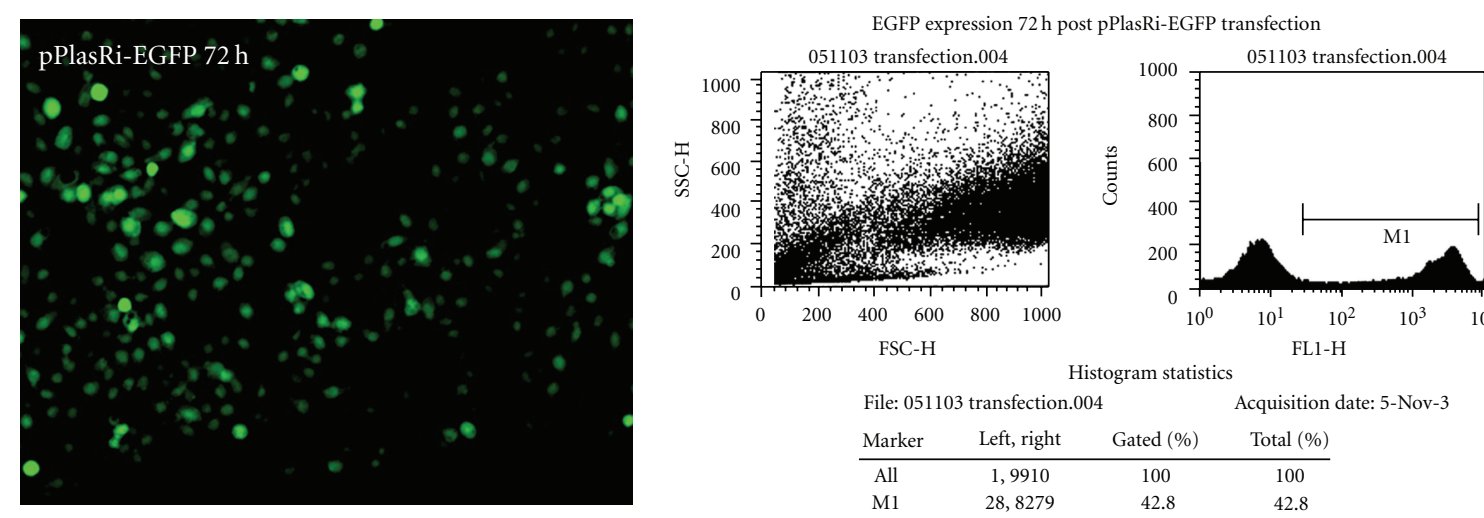

Figure 1: Continued. 

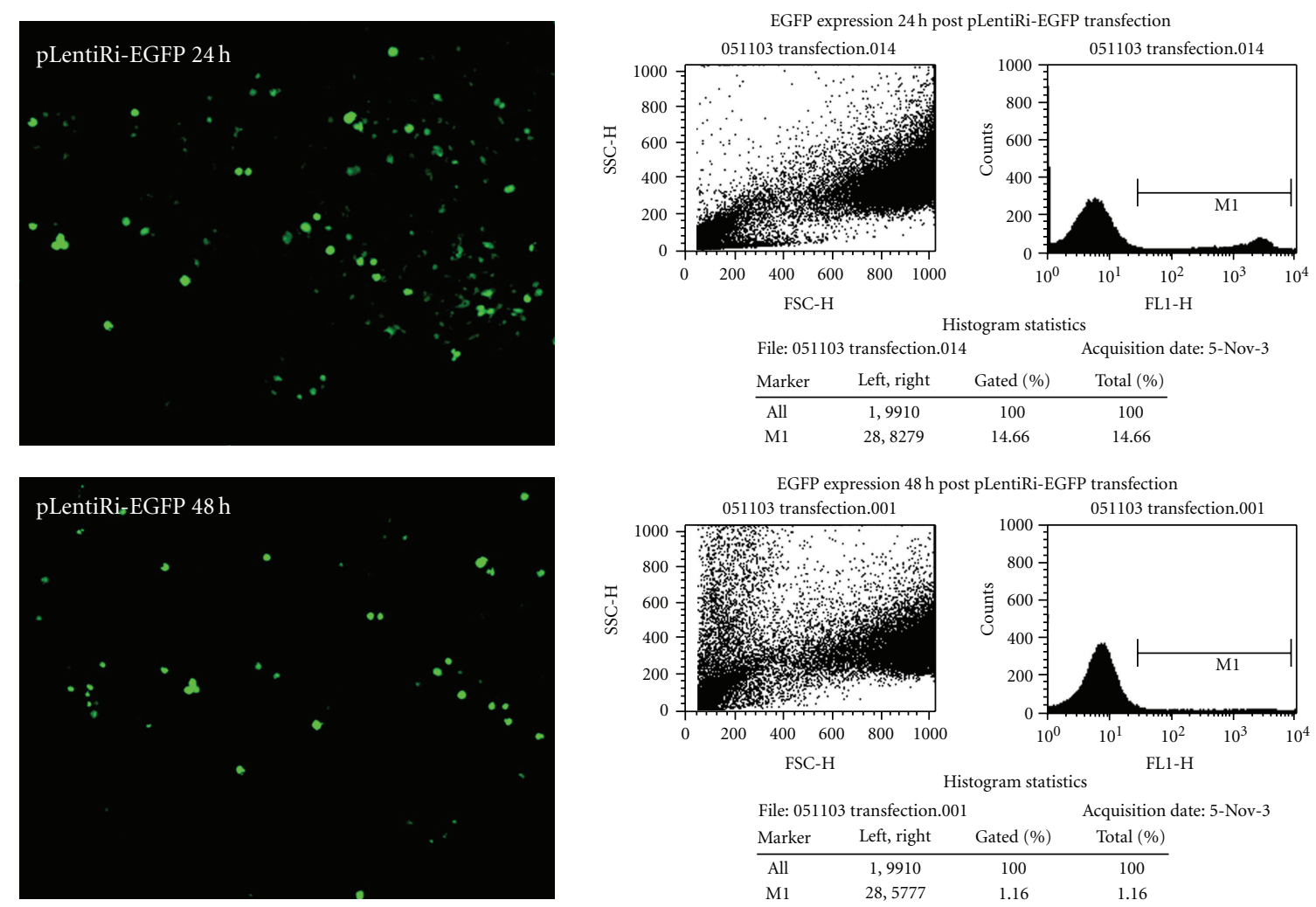

\begin{tabular}{|c|c|c|c|}
\hline \multicolumn{3}{|c|}{ File: 051103 transfection.001 } & \multirow{2}{*}{$\begin{array}{r}\text { Acquisition } \\
\text { Total (\%) }\end{array}$} \\
\hline Marker & Left, right & Gated (\%) & \\
\hline All & 1,9910 & 100 & 100 \\
\hline M1 & 28,5777 & 1.16 & 1.16 \\
\hline
\end{tabular}
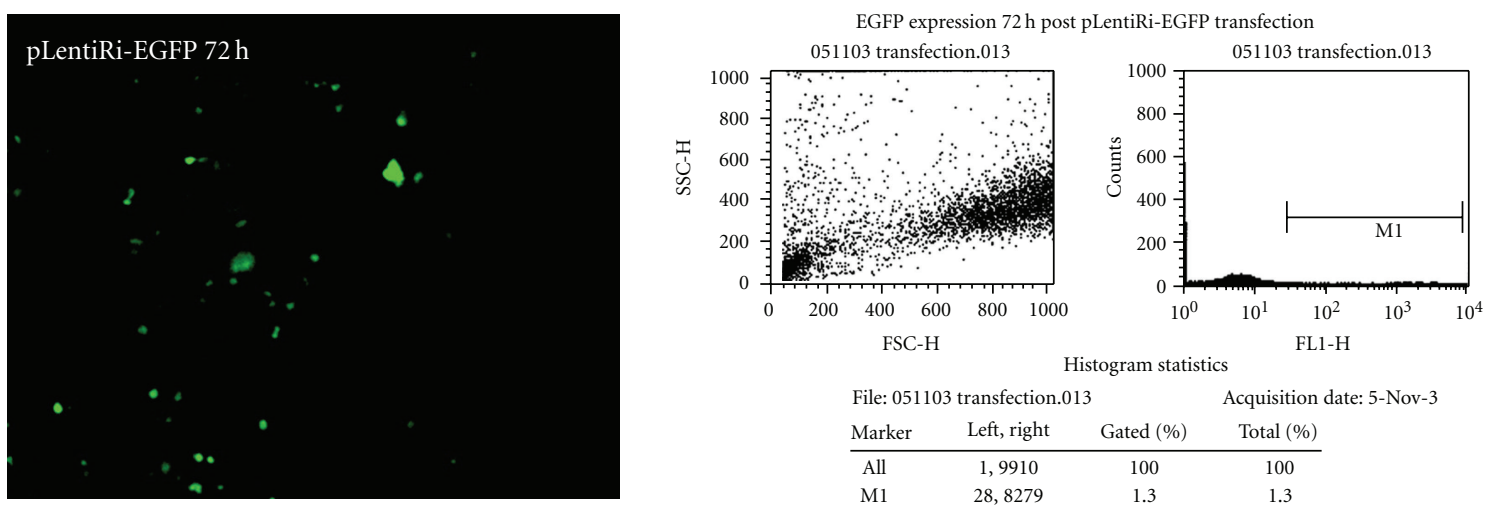

File: 051103 transfection. 013

\begin{tabular}{cccc} 
Marker & Left, right & Gated (\%) & Total (\%) \\
\hline All & 1,9910 & 100 & 100 \\
M1 & 28,8279 & 1.3 & 1.3
\end{tabular}

EGFP gene silencing by RNAi

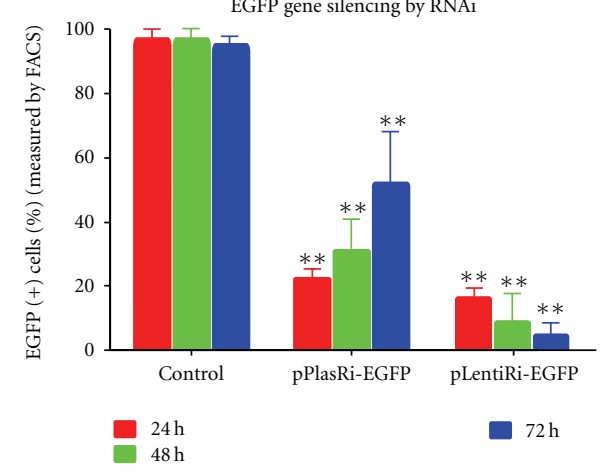

FIGURE 1: Gene silencing of EGFP by transfection of lentiviral and non-lentiviral expression constructs in vitro. RNAi EGFP gene silencing was tested at 24, 48, and $72 \mathrm{hrs}$ in EGFP stably expressing S7 cells post transfection of an EGFP-RNAi expression construct (pPlasRi-EGFP) and a lentiviral RNAi construct (pLentiRi-EGFP). Fluorescence microscope images of cultured cells are demonstrated as well as the FACS analysis. The percent of EGFP positive $(+)$ cells (mean \pm SEM) was assessed using FACS results, and these are shown graphically. There was a significant decrease of EGFP 24 hrs after both transfections compared to controls $(* * P .01$ in comparison with controls). The pPlasRi-EGFP plasmid showed a transient silencing effect in comparison with a persistent inhibition by the pLentiRi-EGFP, which produced a continuing inhibition of EGFP $(+)$ cells at 48 and 72 hrs. Gene silencing of EGFP by the pLentiRi-EGFP plasmid remained at $95.91 \%$ at 72 hrs. 

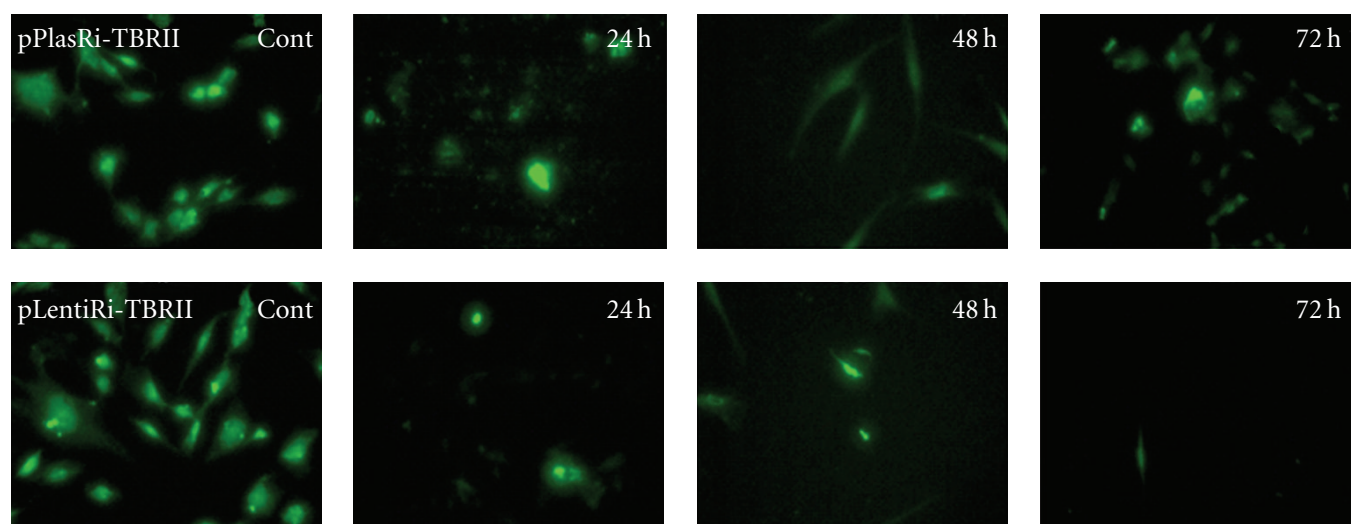

(a)

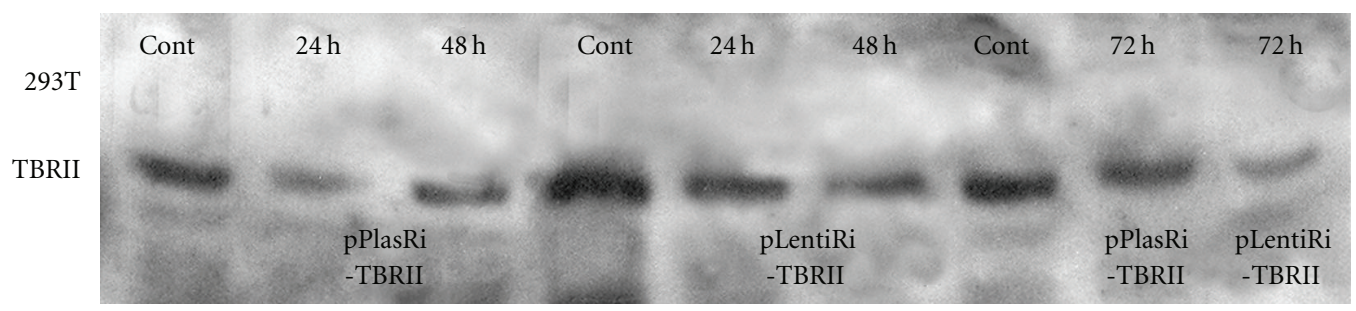

TBRII gene silencing by RNAi in HEK-293T cells

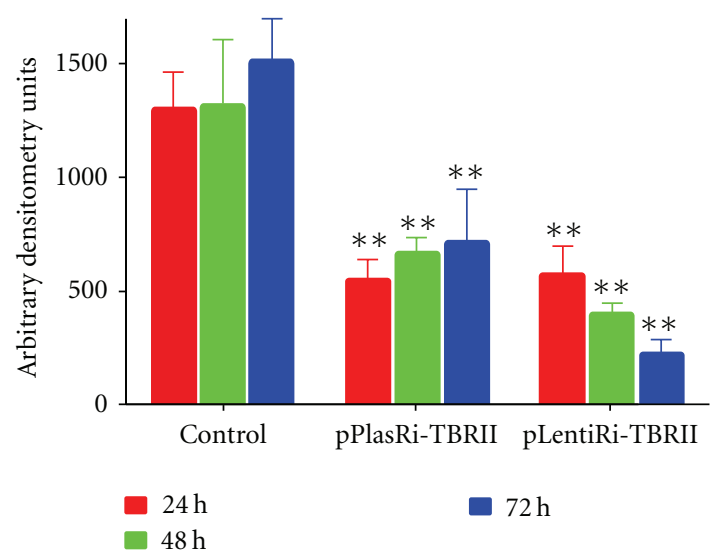

(b)

FIGURE 2: Gene silencing of TBRII by RNAi in HEK-293T cells. pPlasRi-TBRII and pLentiRi-TBRII were transfected into HEK-293T cells. FITC-fluorescence immunohistochemistry of TBRII is demonstrated in the upper two panels. The upper panel demonstrates pPlasRiTBRII and the lower panel demonstrates pLentiRi-TBRII. Both groups showed a decrease of TBRII expression. TBRII was also evaluated by Western blotting (blot panel) and semiquantified by densitometry (graphical presentation). There was significant inhibition of TBRII after both transfections at all time points $\left({ }^{* *} P<.01\right.$ in comparison with controls), but the lentiviral construct increased with time and caused significantly greater silencing at $72 \mathrm{hrs}$ than pPlasRi-TBRII $(P<.01)$.

time points. The lentiviral construct achieved a higher level of inhibition and a longer time of gene silencing of TBRII in both renal epithelial and fibroblast cell lines. These results are demonstrated in Figure 3.

3.6. Inhibition of Fibrogenesis by TBRII RNAi in Renal Cells. The biological effect of TBRII RNAi should be reduced fibrogenesis, represented by reduced ECM production and reduced EMT. Inhibition of fibrogenesis was evaluated by
Western blotting in HEK-293T, NRK-52E, and NRK-49F cells. Collagen III, fibronectin (ECM production), and $\alpha$ SMA expression (EMT) were tested and semiquantified by densitometry at 24,48 , and 72 hours posttransfection of the RNAi expression construct pPlasRi-TBRII and lentiviral RNAi construct pLentiRi-TBRII. These results are demonstrated in Figures 4 and 5. In Figure 4, there was a significant decrease of collagen III expression at 24 and 72 hours post-transfection with both constructs in HEK$293 \mathrm{~T}$ cells, and there was significant inhibition of collagen 

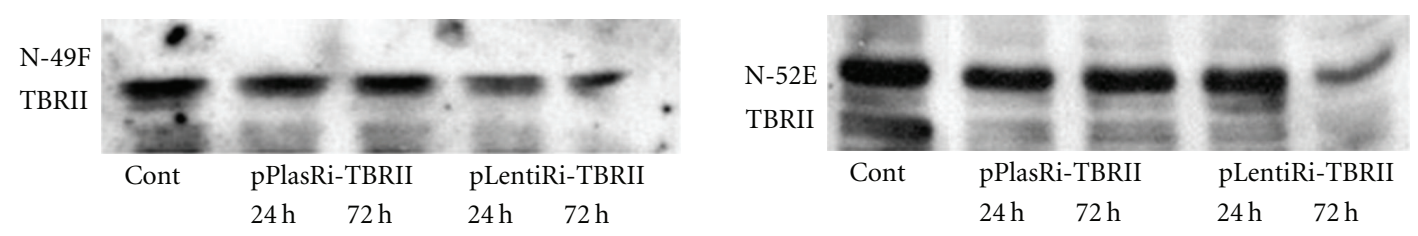

TBRII gene silencing by RNAi in NRK-49F cells

TBRII gene silencing by RNAi in NRK-52E cells
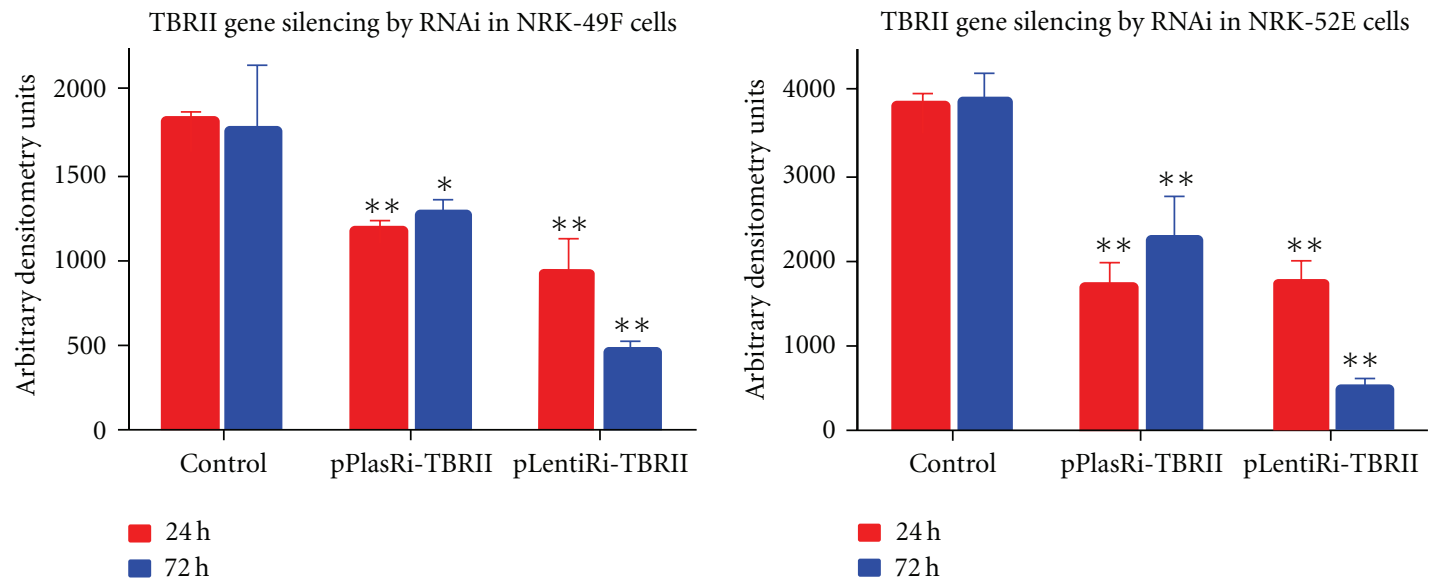

FIGURE 3: Gene silencing of TBRII in renal epithelial and fibroblast cells by RNAi. At 24 and 72 hrs post pPlasRi-TBRII and pLentiRi-TBRII transfection, Western immunoblots for renal fibroblasts (NRK-49F), and epithelial cells (NRK-52E) showed specific inhibition of TBRII. Both RNAi plasmids significantly inhibited TBRII at 24 and 72 hrs post transfection; however, both fibroblast and epithelial cell lines had stronger gene silencing with pLentiRi-TBRII at $72 \mathrm{hrs}\left({ }^{* *} P<.01\right.$ and ${ }^{*} P<.05$, resp., in comparison with controls). Scale bar in each photomicrograph $=100 \mu \mathrm{M}$.

III expression in NRK-52E cells at 24 hours $(P<.01)$. Collagen III inhibition was not apparent at 72 hours in NRK$52 \mathrm{E}$ cells. A significant decrease of collagen III expression was also observed in NRK-49F cell lines by lentiviral RNAi construct at 24 and 72 hours $(P<.01)$ but not in RNAi expression construct transfected cells $(P>.05)$. Fibronectin expression was significantly decreased in HEK-293T, NRK52E, and NRK-49F cells at 24 and 72 hours transfection of either TBRII RNAi expression or lentiviral construct. The variation in responses of the different cell lines, including the time line responses, underlines the complexity of cellular contributions to fibrosis and the antifibrotic responses when planning therapies involving the gene therapies.

There was significantly reduced $\alpha$-SMA expression in NRK-52E and NRK-49F cells post-transfection of both RNAi constructs (Figure 5). Both expression vectors significantly reduced $\alpha$-SMA expression in NRK-49F cells at 72 hours. In the NRK-52E cells, $\alpha$-SMA expression was markedly reduced at both time points, although the reduction in expression was most marked with the pLentiRi vector.

\section{Discussion}

Renal fibrosis is the common pathway of chronic renal disease that progresses to end-stage kidney disease. Chronic renal disease is characterized by persistent accumulation and deposition of ECM which leads to widespread tissue fibrosis $[3,4,24]$. While progress has been made in delineating the cellular and molecular pathogenesis during the last decades, the clinical therapies for chronic renal fibrosis remain extremely limited. Drug delivery systems that take advantage of recent RNAi technologies may open new pathways for therapeutic strategies. In the present study, a lentiviral construct-mediated RNAi system for posttranscriptional, sequence-specific gene silencing of TBRII for the treatment of renal fibrosis was demonstrated. Knockdown of the TBRII gene was observed in cultured renal epithelial and fibroblast cell lines, and consequently EMT and TGF$\beta$ signalling-regulated ECM accumulation was halted. The present study demonstrates that the introduction of TBRII siRNA with lentiviral construct to renal cell populations can effectively suppress the expression of TBRII. If applied in disease, this system could limit renal tubulointerstitial fibrosis.

As a new technology for silencing of a target gene, a pioneer study in the field of renal fibrosis has shown that siRNA targeting TBRII effectively inhibited renal interstitial fibrosis by using an RNAi expression construct with cationic gelatin, in an attempt to overcome the transient, insufficient transfection efficiency of chemically synthesised siRNAs [17]. TBRII expression and $\alpha$-SMA expression were suppressed for up to 10 days in a unilateral ureteral obstruction (UUO) mouse model. A recent study using TGF- $\beta 1$ siRNA showed successful delay of the tubulointerstitial fibrosis process by evaluating collagen accumulation in the UUO model of renal fibrosis, in mice [25]. In this study, an RNAi expression construct processing the U6 RNA promoter, pU6shX, was employed to demonstrate that RNAi targeting of the TGF$\beta$ signalling pathway could be a valuable tool for prevention and treatment of renal tubulointerstitial fibrosis. 


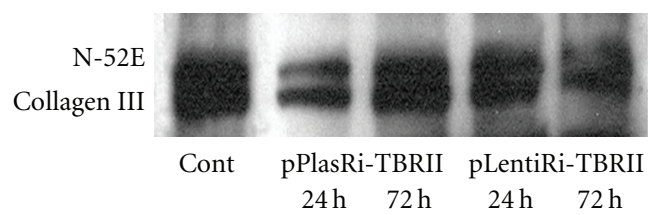

Collagen III inhibition by TBRII-RNAi

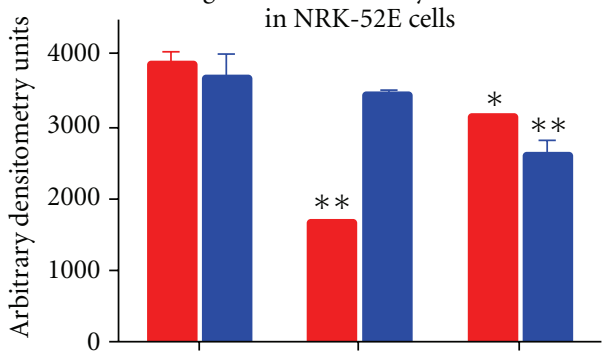

Control pPlasRi-TBRII pLentiRi-TBRII

\section{HEK-293T}

Collagen III

Cont pPlasRi-TBRII Cont Cont pLentiRi-TBRII $24 \mathrm{~h} 72 \mathrm{~h} \quad 24 \mathrm{~h} 72 \mathrm{~h}$

Collagen III inhibition by TBRII-RNAi in HEK-293T cells
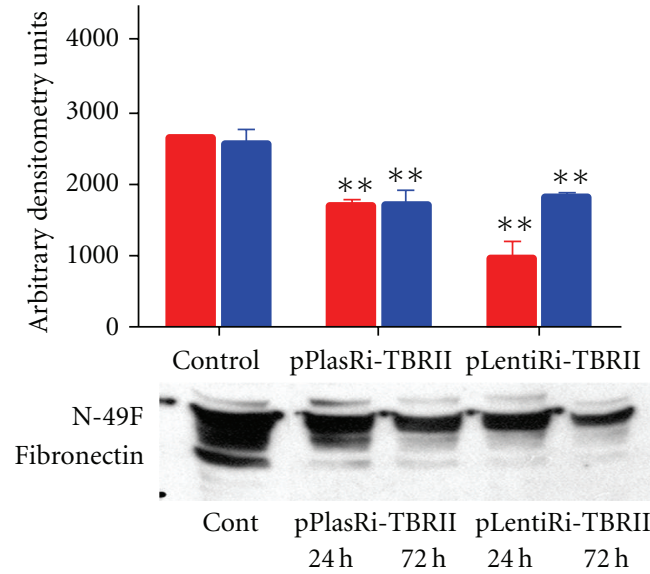

Fibronectin inhibition by TBRII-RNAi

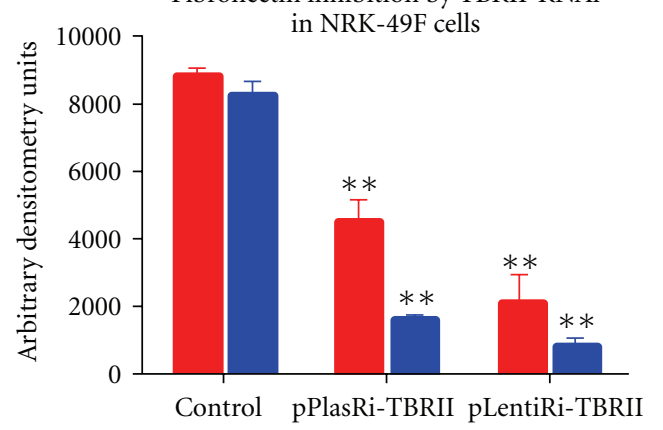

$24 \mathrm{~h}$
$-72 \mathrm{~h}$

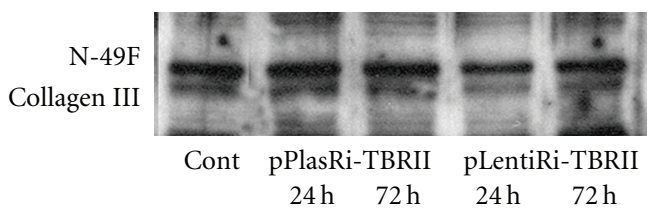

Collagen III inhibition by TBRII-RNAi
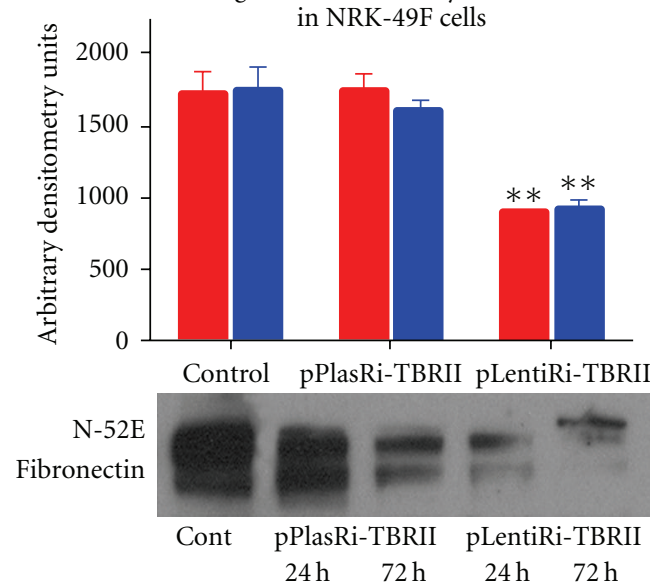

Fibronectin inhibition by TBRII-RNAi in NRK-52E cells
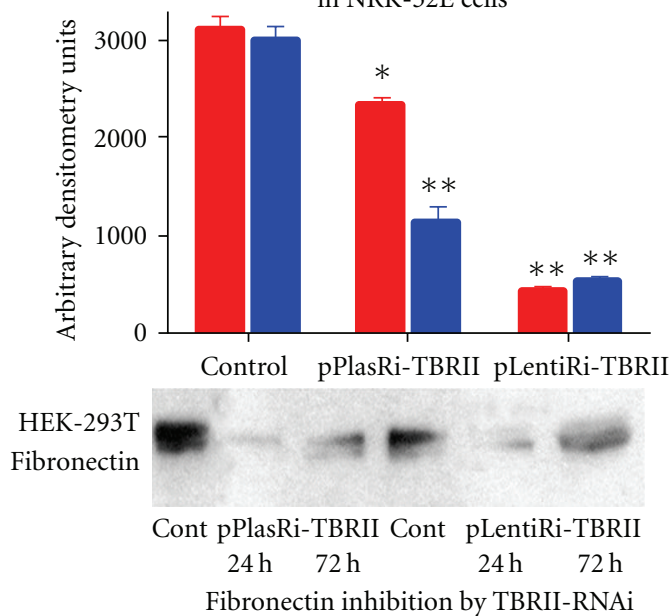

Fibronectin inhibition by TBRII-RNAi in HEK-49F cells

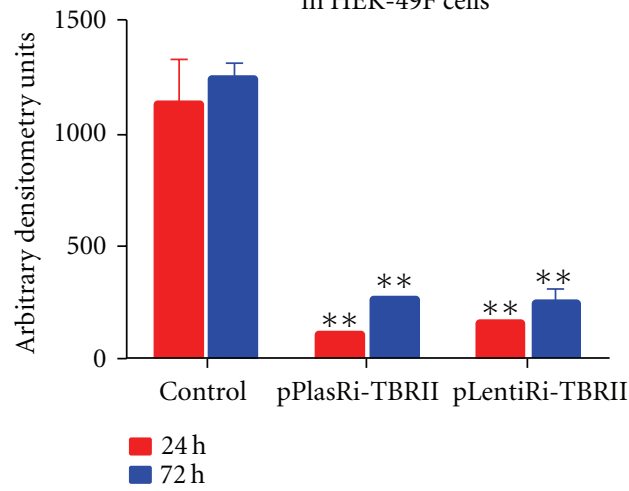

FIGURE 4: Inhibition of ECM by TBRII RNAi in renal cell lines. Western immunoblots of protein extracts from human embryonic kidney cells (HEK-293T), rat renal fibroblasts (NRK-49F), and epithelial cells (NRK-52E) showed significantly reduced ECM production after TBRII RNAi plasmid transfection (fibronectin is abbreviated as "F'nectin" beside the Western immunoblots). There was significantly decreased collagen III and fibronectin expression post-transfection with pPlasRi-TBRII and pLentiRi-TBRII in NRK-49F and NRK-52E cell lines. In comparison with pPlasRi-TBRII, pLentiRi-TBRII produced a stronger and longer inhibitory effect on collagen III in NRK-49F and on fibronectin in all 3 tested cell lines $\left({ }^{* *} P<.01\right.$ and ${ }^{*} P<.05$ in comparison with controls). 

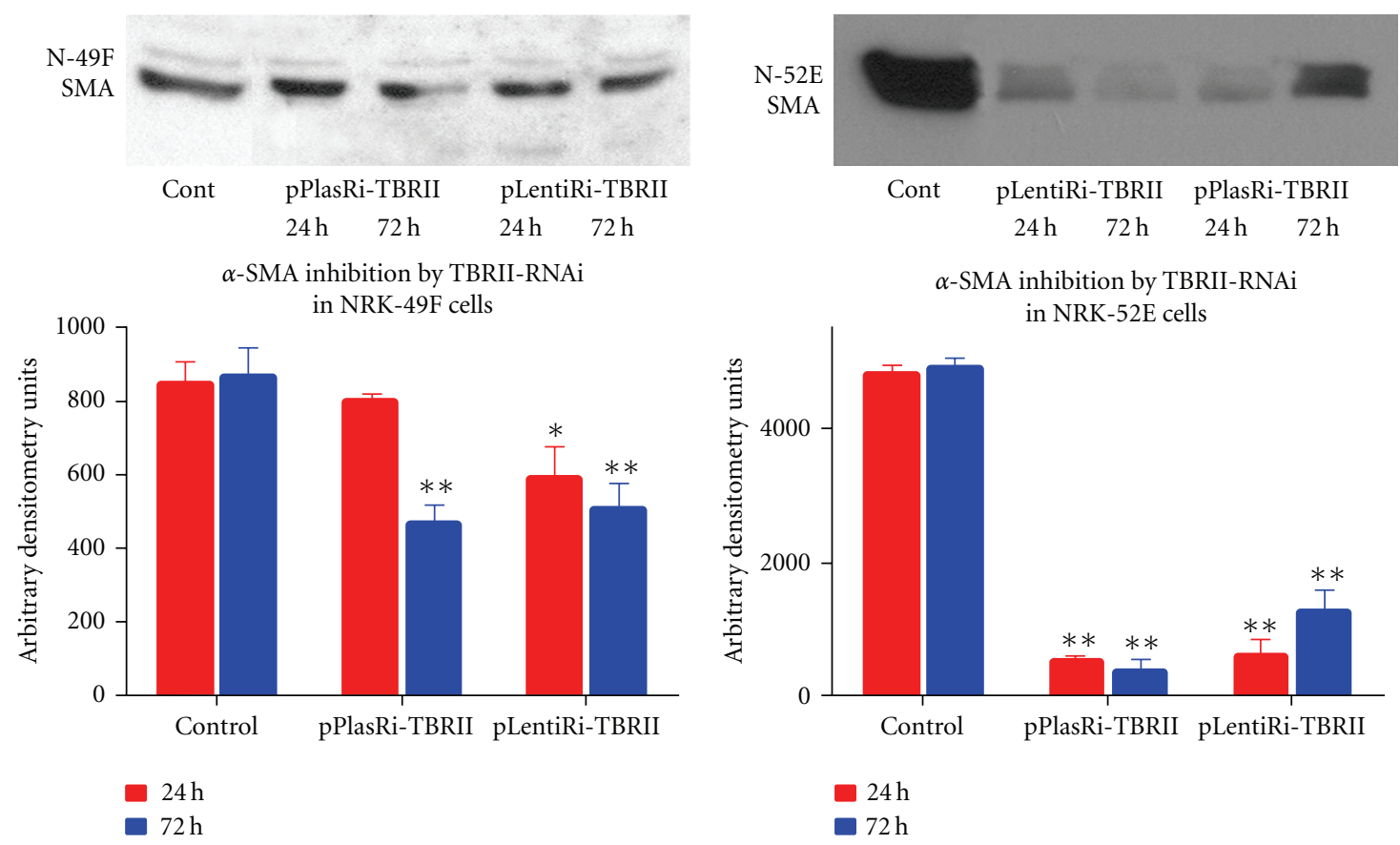

FIGURE 5: Inhibition of $\alpha$-SMA expression by TBRII RNAi in renal cell lines. Both expression vectors significantly reduced $\alpha$-SMA expression in renal fibroblasts (NRK-49F) at 72 hrs indicating successful suppression of myofibroblast activation. In the epithelial cells (NRK-52E), $\alpha$ SMA expression was markedly reduced with both expression constructs at both time points, indicating inhibition of EMT. The reduction in expression was most marked with the lentiviral vector. ADU: arbitrary densitometry units.

In the present study, a similar RNAi construct, namely, pPlasRi, was used with the polymerase III H1-RNA gene promoter $[23,25,26]$. It produces a small RNA transcript lacking a polyadenosine tail and has a well-defined start of transcription and a termination signal consisting of five thymidines in a row [26]. Two pairs of 19-nt sequence-targeted short hairpin RNAi (shRNAi) cassettes were designed and cloned into the RNAi expression construct. EGFP was selected as the experimental control because fluorescence is easy to observe under the fluorescent microscope and quantified by FACS analysis $[27,28]$. The result showed satisfactory EGFP suppression of $78.28 \%$ at 24 hours. The inhibition effect was however weakened to $69.37 \%$ and $40.09 \%$ at 48 hours and 72 hours, respectively, post transfection. This indicated that a gene silencing effect of EGFP could be achieved by an RNAi expression construct, but the effect is transient. Similarly, after construction of the TBRII RNAi expression construct and its transfection to HEK-293T cell lines, reduced TBRII expression was most pronounced at 24 hours post transfection. In comparison with 48 hours and 72 hours, the transient RNAi effect of the expression construct was again noted.

In principle, there are two general siRNA delivery methods. One uses chemically synthesised 19-21nt siRNA with apparent short-lived effect [28]. The other approach is vector-based siRNA, including the use of plasmid vectors and viral vectors. Vector-based siRNA has a more prolonged effect and is relatively inexpensive. Plasmid-mediated biological activity of RNAi still needs to be improved by various means. Several synthetic materials, including cationic liposomes, poly-L-lysine, and PEI, have been molecularly designed to allow successful transfection of plasmid DNA for mammalian cells both in vitro and in vivo. In Kushibiki et al.'s pSUPER TBRII RNAi system, cationic gelatin was used to facilitate transfection, and it showed relatively satisfactory gene silencing effect in the UUO model for up to 10 days [5]. Our previous experience indicated that a cationic polymer PEI could facilitate better transfection than poly-L-lysine [29], and this was confirmed in the renal cell lines tested. The results showed that there was RNAi effect on EGFP and TBRII in both renal epithelial and fibroblast cells after RNAi expression plasmid transfection.

A prerequisite for developing RNAi-based therapeutics is an efficient delivery vehicle to transfer the RNAi cassette into the target cells. Our results demonstrated the transient nature of the RNAi vectors and the ability of the lentiviral vector-mediated RNAi system for a sustained transduction effect, as had been demonstrated previously in terminally differentiated cells [30]. The advantages of lentivirus for gene transfer, especially to non-dividing cells, make the RNAi technique more accessible for specific in vitro and in vivo silencing in renal cell populations. The lentiviral construct showed significant silencing of EGFP in S7 cells. There was 84.17\%, 91.57\%, and 95.91\% decrease of EGFP achieved at 24 hours, 48 hours, and 72 hours post PEI facilitated transfection. In comparison with the non-lentiviral RNAi expression construct, the gene-silencing effect of the lentiviral RNAi construct was stronger and persistent $(P<.01)$. TBRII gene silencing in HEK-293T cells showed a similar result that favoured the lentiviral RNAi construct. 
TBRII expression, ECM production (collagen III, fibronectin), and marker of EMT ( $\alpha$-SMA expression) were examined after gene suppression of TBRII. Both constructs achieved significant inhibition with reduced collagen III and fibronectin expression in HEK-293T, NRK-49F, and NRK-52E cells, and with the lentiviral RNAi construct producing a more robust inhibition of fibrogenesis and continuing inhibition of TBRII 72 hours post-transfection. Complete TBRII silencing was not achieved, even though a lentiviral construct was employed, and was facilitated by a cationic transfection aid PEI. During renal fibrogenesis in vivo, several events occur in parallel, including an influx of monocytes/macrophages, activation of myofibroblasts, accumulation of various ECM proteins, and EMT [31,32]. As an indicator of fibroblast activation and tubular EMT, $\alpha$-SMA expression was evaluated in renal fibroblasts and epithelial cells. Significant reduction of $\alpha$-SMA was shown by Western blot analysis, indicating an inhibitory effect on fibroblast activation and EMT.

\section{Conclusion}

The present data demonstrated that the lentiviral-mediated RNAi in renal cell populations was effective not only in silencing TBRII gene, therefore, blocking TGF- $\beta$ signalling, but also modulating activation of fibroblasts, development of EMT, and reducing ECM accumulation. Successful prolonged presence of RNAi in renal cells in vitro was also demonstrated. The lentiviral RNAi transfection facilitated by cationic polymer PEI was superior to the classical RNApromoter-possessed plasmid-mediated RNAi. Thus, this technique of delivering RNAi into tubulointerstitial cells could be a practical therapeutic method for progressive renal interstitial fibrosis.

\section{List of Abbreviations}

ANOVA: Analysis of variance

BSA: Bovine serum albumin

DMEM: Dulbecco's modified Eagle's medium

dsRNA: Double-stranded RNA

ECM: Extracellular matrix

EGFP: Enhanced green fluorescent protein

EMT: Epithelial-to-mesenchymal transition

F'nectin: Fibronectin

$\gamma$-GT: $\quad$ Gamma-glutamyl transpeptidase

HEK: Human embryonic kidney

NRK: Normal rat kidney

PCR: Polymerase chain reaction

PEI: Polyethyleneimine

RNAi: RNA interference

SF: $\quad$ Serum-free

siRNA: Silencing RNA

TBRII: Transforming growth factor-beta receptor II

TBST: Tris-buffered saline-Tween

TGF- $\beta$ : Transforming growth factor-beta.

\section{Acknowledgments}

For this work, T. Yang was supported by an International Postgraduate Research Scholarship from The University of Queensland and an Australian Postgraduate Award from the Australian Federal Government. The paper was also supported by a grant from Kidney Health Australia. All authors contributed to the development of the testable hypotheses, analysis of data, development of results concepts, and writing the paper. Most of the cell culture lentiviral work was carried out by Dr. T. Yang, with some help from Drs. B. K. Pat and B. Zhang.

\section{References}

[1] M. Zeisberg, F. Strutz, and G. A. Müller, "Renal fibrosis: an update," Current Opinion in Nephrology and Hypertension, vol. 10, no. 3, pp. 315-320, 2001.

[2] A. A. Eddy, "Can renal fibrosis be reversed?" Pediatric Nephrology, vol. 20, no. 10, pp. 1369-1375, 2005.

[3] A. A. Eddy, "Progression in chronic kidney disease," Advances in Chronic Kidney Disease, vol. 12, pp. 353-365, 2005.

[4] Y. Liu, "Renal fibrosis: new insights into the pathogenesis and therapeutics," Kidney International, vol. 69, no. 2, pp. 213-217, 2006.

[5] T. Kushibiki, N. Nagata-Nakajima, M. Sugai, A. Shimizu, and Y. Tabata, "Delivery of plasmid DNA expressing small interference RNA for TGF- $\beta$ type II receptor by cationized gelatin to prevent interstitial renal fibrosis," Journal of Controlled Release, vol. 105, no. 3, pp. 318-331, 2005.

[6] X. R. Huang, A. C. K. Chung, X. J. Wang, K. N. Lai, and H. Y. Lan, "Mice overexpressing latent TGF- $\beta 1$ are protected against renal fibrosis in obstructive kidney disease," American Journal of Physiology, vol. 295, no. 1, pp. F118-F127, 2008.

[7] A. L. Attisano, J. L. Wrana, F. Lopez-Casillas, and J. Massague, "TGF- $\beta$ receptors and actions," Biochimica et Biophysica Acta, vol. 1222, no. 1, pp. 71-80, 1994.

[8] J. L. Wrana, L. Attisano, R. Wieser, F. Ventura, and J. Massagué, "Mechanism of activation of the TGF- $\beta$ receptor," Nature, vol. 370, no. 6488, pp. 341-347, 1994.

[9] A. Nakao, T. Imamura, S. Souchelnytskyi et al., "TGF- $\beta$ receptor-mediated signalling through $S \operatorname{mad} 2, \operatorname{Smad} 3$ and Smad4," EMBO Journal, vol. 16, no. 17, pp. 5353-5362, 1997.

[10] P. Lavoie, G. Robitaille, M. Agharazii, S. Ledbetter, M. Lebel, and R. Larivière, "Neutralization of transforming growth factor- $\beta$ attenuates hypertension and prevents renal injury in uremic rats," Journal of Hypertension, vol. 23, no. 10, pp. 18951903, 2005.

[11] A. Benigni, C. Zoja, D. Corna et al., "Add-on anti-TGF$\beta$ antibody to ACE inhibitor arrests progressive diabetic nephropathy in the rat," Journal of the American Society of Nephrology, vol. 14, no. 7, pp. 1816-1824, 2003.

[12] W. A. Border, N. A. Noble, T. Yamamoto et al., "Natural inhibitor of transforming growth factor- $\beta$ protects against scarring in experimental kidney disease," Nature, vol. 360, no. 6402, pp. 361-364, 1992.

[13] F. Wu, H. Yao, A. Bader et al., "Decorin gene transfer inhibited the expression of TGF $\beta 1$ and ECM in rat mesangial cells," European Journal of Medical Research, vol. 12, no. 8, pp. 360368, 2007. 
[14] Y. Isaka, Y. Akagi, Y. Ando et al., "Gene therapy by transforming growth factor- $\beta$ receptor-IgG Fc chimera suppressed extracellular matrix accumulation in experimental glomerulonephritis," Kidney International, vol. 55, no. 2, pp. 465-475, 1999.

[15] Y. S. Haviv, K. Takayama, P. A. Nagi et al., "Modulation of renal glomerular disease using remote delivery of adenoviralencoded soluble type II TGF- $\beta$ receptor fusion molecule," Journal of Gene Medicine, vol. 5, no. 10, pp. 839-851, 2003.

[16] M. Nakamuta, S. Morizono, S. Tsuruta, M. Kohjima, K. Kotoh, and M. Enjoji, "Remote delivery and expression of soluble type II TGF-beta receptor in muscle prevents hepatic fibrosis in rats," International Journal of Molecular Medicine, vol. 16, no. 1, pp. 59-64, 2005.

[17] T. Kushibiki, N. Nagata-Nakajima, M. Sugai, A. Shimizu, and Y. Tabata, "Targeting of plasmid DNA to renal interstitial fibroblasts by cationized gelatin," Biological and Pharmaceutical Bulletin, vol. 28, no. 10, pp. 2007-2010, 2005.

[18] A. M. Gressner and R. Weiskirchen, "The tightrope of therapeutic suppression of active transforming growth factor$\beta$ : high enough to fall deeply?" Journal of Hepatology, vol. 39, no. 5, pp. 856-859, 2003.

[19] L. Naldini, U. Blomer, F. H. Gage, D. Trono, and I. M. Verma, "Efficient transfer, integration, and sustained longterm expression of the transgene in adult rat brains injected with a lentiviral vector," Proceedings of the National Academy of Sciences of the United States of America, vol. 93, no. 21, pp. 11382-11388, 1996.

[20] M. Sioud, "Targeted delivery of antisense oligonucleotides and siRNAs into mammalian cells," Methods in Molecular Biology, vol. 487, pp. 61-82, 2009.

[21] G. L. Gusella, E. Fedorova, B. Hanss, D. Marras, M. E. Klotman, and P. E. Klotman, "Lentiviral gene transduction of kidney," Human Gene Therapy, vol. 13, no. 3, pp. 407-414, 2002.

[22] H. Y. Lin, X.-F. Wang, E. Ng-Eaton, R. A. Weinberg, and H. F. Lodish, "Expression cloning of the TGF- $\beta$ type II receptor, a functional transmembrane serine/threonine kinase," Cell, vol. 68, no. 4, pp. 775-785, 1992, Erratum in Cell, vol. 70, no. 6, pp. 1069, 1992.

[23] J.-Y. Yu, S. L. DeRuiter, and D. L. Turner, "RNA interference by expression of short-interfering RNAs and hairpin RNAs in mammalian cells," Proceedings of the National Academy of Sciences of the United States of America, vol. 99, no. 9, pp. 60476052, 2002.

[24] M. Zeisberg, Y. Maeshima, B. Mosterman, and R. Kalluri, "Renal fibrosis: extracellular matrix microenvironment regulates migratory behavior of activated tubular epithelial cells," American Journal of Pathology, vol. 160, no. 6, pp. 2001-2008, 2002.

[25] M. Hwang, H.-J. Kim, H.-J. Noh et al., "TGF- $\beta 1$ siRNA suppresses the tubulointerstitial fibrosis in the kidney of ureteral obstruction," Experimental and Molecular Pathology, vol. 81, no. 1, pp. 48-54, 2006.

[26] T. R. Brummelkamp, R. Bernards, and R. Agami, "A system for stable expression of short interfering RNAs in mammalian cells," Science, vol. 296, no. 5567, pp. 550-553, 2002.

[27] O. Donzé and D. Picard, "RNA interference in mammalian cells using siRNAs synthesized with T7 RNA polymerase," Nucleic Acids Research, vol. 30, no. 10, p. e46, 2002.

[28] S. M. Elbashir, W. Lendeckel, and T. Tuschl, "RNA interference is mediated by 21- and 22-nucleotide RNAs," Genes and Development, vol. 15, no. 2, pp. 188-200, 2001.
[29] B. Zhang, H. Q. Xia, G. Cleghorn, G. Gobe, M. West, and M. Q. Wei, "A highly efficient and consistent method for harvesting large volumes of high-titre lentiviral vectors," Gene Therapy, vol. 8, no. 22, pp. 1745-1751, 2001.

[30] T. Abbas-Terki, W. Blanco-Bose, N. Déglon, W. Pralong, and P. Aebischer, "Lentiviral-mediated RNA interference," Human Gene Therapy, vol. 13, no. 18, pp. 2197-2201, 2002.

[31] M. Eikmans, J. J. Baelde, E. de Heer, and J. A. Bruijn, "ECM homeostasis in renal diseases: a genomic approach," Journal of Pathology, vol. 200, no. 4, pp. 526-536, 2003.

[32] M. Iwano and E. G. Neilson, "Mechanisms of tubulointerstitial fibrosis," Current Opinion in Nephrology and Hypertension, vol. 13, no. 3, pp. 279-284, 2004. 

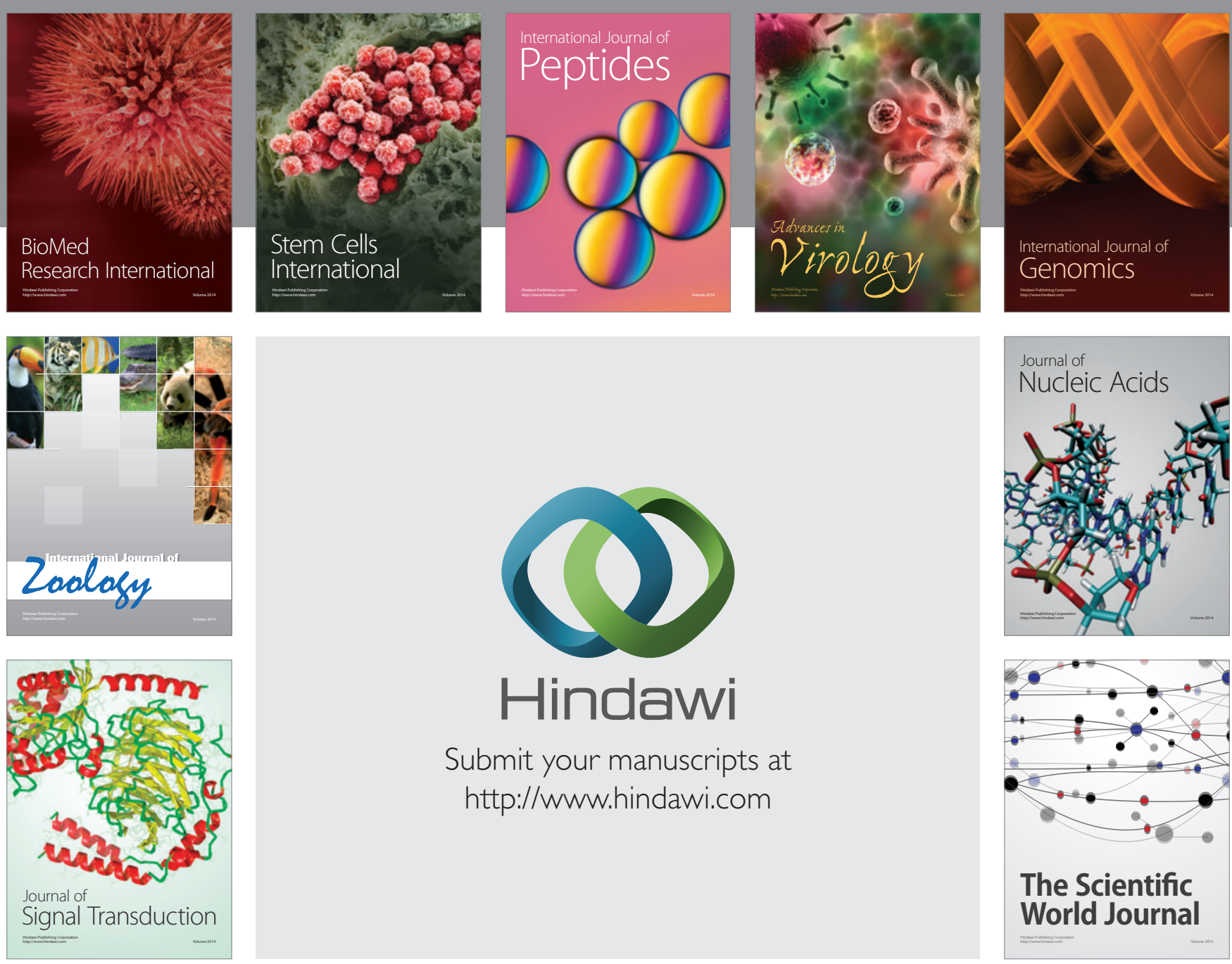

Submit your manuscripts at

http://www.hindawi.com
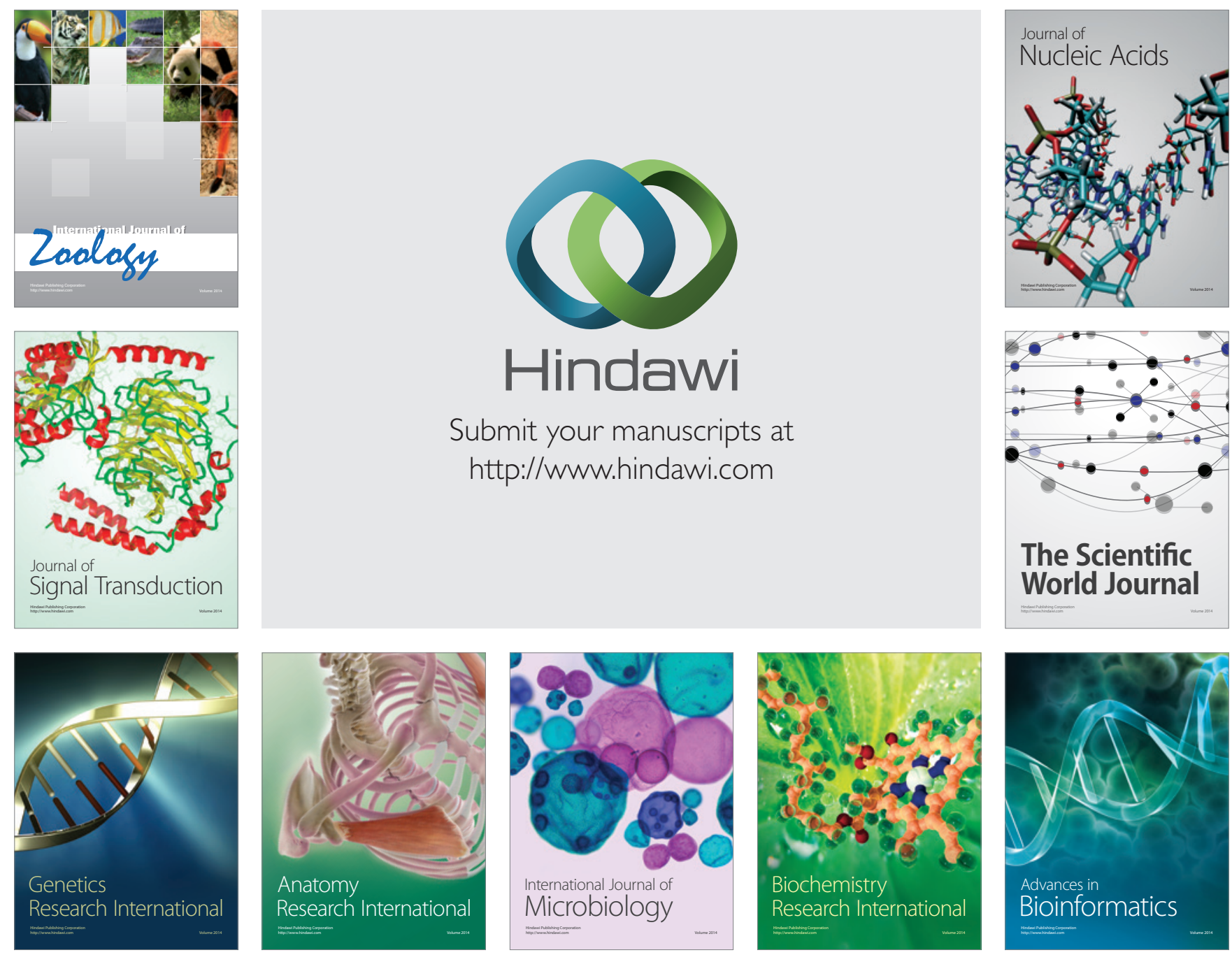

The Scientific World Journal
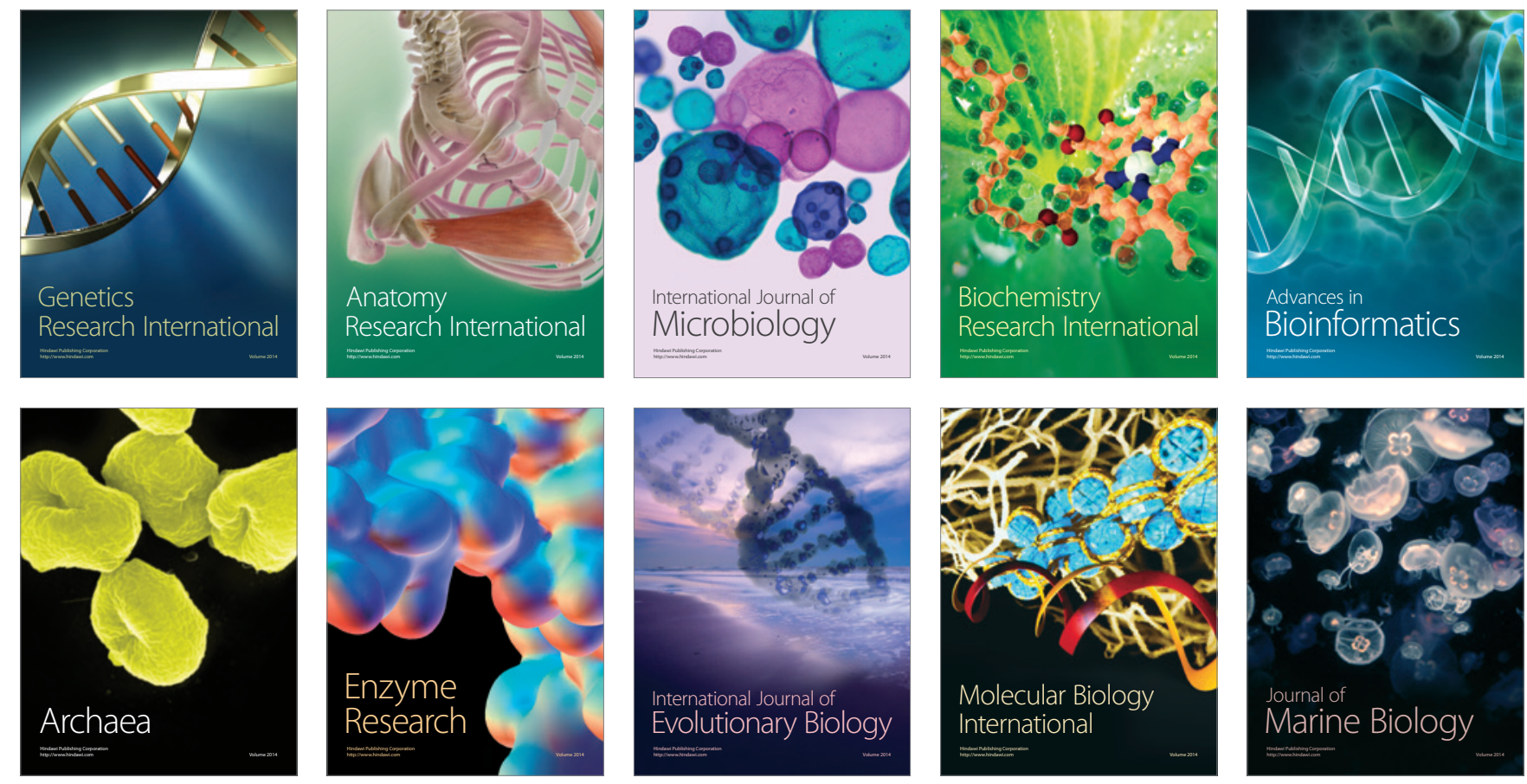